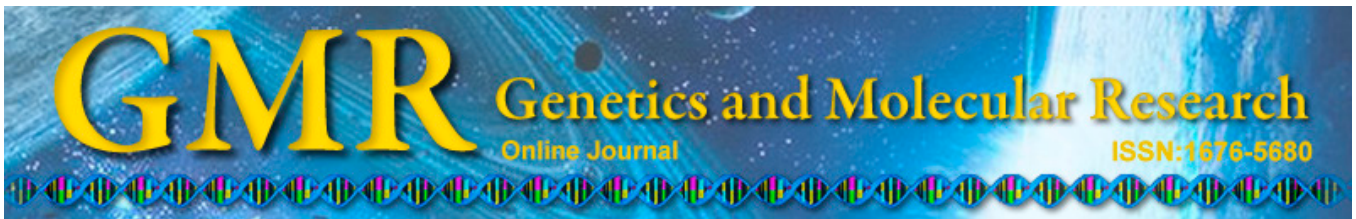

\title{
Expression of EpCAM and Wnt/ $\beta$-catenin in human colon cancer
}

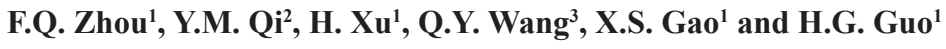 \\ ${ }^{1}$ Department of General Surgery, Shandong Province Binzhou People's Hospital, \\ Binzhou, Shandong, China \\ ${ }^{2}$ Department of Gastroenterology, Shandong Province Binzhou People's Hospital, \\ Binzhou, Shandong, China \\ ${ }^{3}$ Department of Science and Education, \\ Shandong Province Binzhou People's Hospital, Binzhou, Shandong, China \\ Corresponding author: F.Q. Zhou \\ E-mail: zhoufengqiang2014@163.com
}

Genet. Mol. Res. 14 (2): 4485-4494 (2015)

Received June 26, 2014

Accepted October 20, 2014

Published May 4, 2015

DOI http://dx.doi.org/10.4238/2015.May.4.6

ABSTRACT. The aims of this study were to explore the correlation
between the expression of EpCAM and the Wnt/ $\beta$-catenin pathway
in human colon cancer and its clinical significance for the evaluation
of cancer prognosis. Samples from colon cancer, para-carcinoma, or
benign intestinal tissue from individual patients (50) and from normal
intestinal mucosal tissues (20) were obtained from the Pathology
Department of the Shandong Province Binzhou People's Hospital
(Shandong, China). Immunohistochemistry was used to detect the
expression levels of EpCAM and $\beta$-catenin proteins in these tissues,
and the prognoses of the patients from whom the samples were derived
were determined on follow-up examination. The corresponding in vitro
mechanistic siRNA experiments were subsequently performed in the
human colon cancer cell line HCT116 to observe the regulatory effects
of silencing EpCAM expression on the Wnt/ $\beta$-catenin pathway. From
these analyses, we determined that the expression levels of EpCAM
and $\beta$-catenin were higher in cancer tissues compared with other tissues 
from the same patient, and that the expression of EpCAM and Wnt $/ \beta$ catenin in colon cancers were positively correlated. The prognostic analysis showed an inverse correlation between EpCAM and Wnt/ $\beta$ catenin expression and patient prognosis. A further examination of cellular mechanisms confirmed that the silencing of EpCAM led to decreased expression of $\mathrm{Wnt} / \beta$-catenin, and thus reduced proliferation and increased the apoptosis ratio in the cells. These results suggest that suppression of EpCAM might be a new approach for treating colon cancer.

Key words: EpCAM; Wnt/ $\beta$-catenin; Colon cancer

\section{INTRODUCTION}

The epithelial cell adhesion protein (EpCAM) is a member of the adhesion molecule family. EpCAM is involved in regulating the functions of cell adhesion, migration, proliferation and differentiation (Clevers and Nusse, 2012). The molecule characteristically shows little to no expression in normal tissues and high expression in tumor tissues (Carmon et al., 2011). Currently, the role of EpCAM in the etiology, development, and prognosis of cancer is not clear. In this study, the relationship between EpCAM and the currently accepted Wnt/ $\beta$-catenin pathway in tumorigenesis and cancer development was explored by retrospective analysis of pathological data from our hospital, and the suggested mechanisms verified by in vitro cell culture experiments, as reported below.

\section{MATERIAL AND METHODS}

\section{Clinical data}

Patients who underwent radical operations for colon cancer or various colon resection operations in the general surgery department of the Shandong Province Binzhou People's Hospital (Shandong, China) between 2000 and 2008 were selected for participation in this study. The patients had not received preoperative chemoradiation therapy or other immune therapies. The postoperative survival time was counted from the date of operation to the date of last follow-up (June, 2013) or death. Colon cancer, para-carcinoma, or benign intestinal tissue samples were collected from 50 patients, in addition to 20 samples from normal intestinal mucosal tissues. The pre-operative diagnosis of colon cancer was definitively diagnosed by postoperative pathological examination for all 50 patients. The samples harvested included colon cancer tissue, diagnosed definitively by pathology, and para-carcinoma tissue, which were defined as intestinal mucosal tissue with a distance of $5 \mathrm{~cm}$ outside of the edges of the region containing cancer-like cells. The benign intestinal tissues were from non-neoplastic lesions such as ulcerative colitis and Crohn's disease. The normal intestinal mucosa samples were collected from nonintestinal lesions such as accidental trauma. The protocol of this study was approved by the Shandong Province Binzhou People's Hospital (Shandong, China). Informed consents were obtained from all subjects. 


\section{Clinical follow-up and data statistics}

The clinically and pathologically relevant patient information was recorded and the cases were followed up; this included the patients' surgical staging (stage I-IV); pathological staging (low, medium, or high), postoperative survival time, tumor recurrence, and metastasis rate. Using these, the different expression levels of EpCAM and $\beta$-catenin in the various tissues, and their correlations with patient prognoses, were statistically analyzed.

\section{Immunohistochemical detection}

The human colon cancer, para-carcinoma, or benign intestinal tissues, and normal intestinal mucosal tissue received by the Pathology Department, were fixed with $4 \%$ paraformaldehyde immediately after routine specimen collection, and were processed into paraffin sections and preserved. In this study, the relevant specimen sections were incubated with $3 \%$ hydrogen peroxide solution for $15 \mathrm{~min}$ after the dewaxing treatment to eliminate endogenous peroxidase. After being rinsed with distilled water, antigen retrieval and blocking of protein impurities were carried out for EpCAM and $\beta$-catenin, respectively.

Blocked tissue sections were incubated overnight at $4^{\circ} \mathrm{C}$, in a $1: 100$ dilution of the primary antibody EpCAM (MAB960) and $\beta$-catenin (MAB13291) monoclonal antibodies (R \& D Systems, Minneapolis, MN, USA). After washing with PBS, the tissue sections were incubated with biotin-labeled secondary antibodies for $15 \mathrm{~min}$ and labeled with horseradish peroxidase, and then were stained with Dolichos biflorus agglutinin (DBA) and restained with hematoxylin. After graded alcohol dehydration, the sections were mounted. For this analysis, the positively stained cancerous tissue was taken as a positive control, and the normal tissue incubated with PBS instead of primary antibody was taken as the negative control. A semi-quantitative assessment of staining results was carried out according to observed staining intensity as follows: EpCAM-positive cells were identified by the appearance of granular cytoplasm, and assessment was performed according to the criteria of Spizzo et al. (2011). $\beta$-Catenin positive cells were identified by positively stained envelopes, and the assessment for these was performed according to method of Jawhari et al. (1999). For ease of assessment, the semi-quantitative assessment method for both positive and negative indices was that when the positive cells were at $\leq 150 \%$ of the total cells, they were considered as weakly positive; and when no positive cells could be detected, they were considered as negative.

\section{Cell culture and EpCAM silencing}

The human colon cancer cell line HCT116 was selected for use in this study. Cells were cultured with DMEM high glucose medium containing 10\% fetal bovine serum (Gibco, Gaithersburg, MD, USA) at $37^{\circ} \mathrm{C}$ in the $5 \% \mathrm{CO}_{2}$ incubator. Subculturing was performed when cells grew to $90 \%$ confluence (average 2-3 days), and the experiment was started at $24 \mathrm{~h}$ after passage when the cells were in the exponential growth phase.

The design process of the EpCAM siRNA was as follows: the complete genetic sequence of EpCAM was identified in GenBank (NM_002354.2), and the Ambion siRNA design software (Ambion, Austin, TX, USA) was used for preliminary design, with subsequent screening using BLAST (National Institutes of Health, USA; http://blast.ncbi.nlm.nih.gov/ Blast.cgi) for elimination of siRNAs with homologies to non-targeted genes. RNAstructure 
4.4 software (http://rna.chem.rochester.edu/) was used for secondary structure analysis and screening. The specific sequences of the final selected target and negative control siRNAs were as follows: EpCAM-726-siRNA sense: 5'-GGG UCA GAA GAA CAG ACA ATT-3'; Antisense: 5'-UUU CUG UUC UUC UGA CCC TT-3'.

The cells were transferred to a 24 -well culture plate and allowed to grow to $70 \%$ confluence, and then siRNA transfection was carried out using the Liposome siRNA transfection reagent kit Lipofectamine@2000 (Code: 11668-019-1.5, Invitrogen, Grand Island, NY, USA). Specific steps were performed in accordance with manufacturer instructions. The transfection efficiency was evaluated at $48 \mathrm{~h}$ after transfection.

Transfection efficiency was detected as follows: fluorescent liposomes carrier was transfected according to the manual. After $48 \mathrm{~h}$, three separate vision fields were randomly selected to calculate transfection efficiency (the ratio of fluorescent cells to total cells). The following evaluation would be performed if the transfection efficiency over $90 \%$.

\section{Analysis of gene expression after transfection}

Changes in gene expression after transfection were detected using reverse transcription polymerase chain reaction (RT-PCR) and Western blot.

For RT-PCR, the cells were allowed to grow to greater than $90 \%$ confluence in good condition. After the medium was decanted, the cells were washed with precooled PBS, and intracellular total RNA was extracted using TRIzol (Takara Bio., Otsu, Shiga, Japan), with reference to the operating manual for specific extraction methodology. After preliminary extraction, RNasin and DNaseI were used to eliminate genomic DNA, with confirmation by spectrophotometry, and RNA concentrations and purities were confirmed to meet experimental requirements. The RT-PCR Kit (Takara, D9108A) was used for reverse transcription, and gene expression measured by detection of the cDNA formed after reverse transcription by fluorescent quantitative PCR. The cycling conditions consisted of 40 cycles of $30 \mathrm{~s}$ at $95^{\circ} \mathrm{C}$, $30 \mathrm{~s}$ at $56^{\circ} \mathrm{C}$, and $30 \mathrm{~s}$ at $72^{\circ} \mathrm{C}$. PCR amplifications were performed in three duplicates for each sample. Gene expression levels were quantified relative to the expression of $\beta$-actin using an optimized comparative $\mathrm{Ct}(\Delta \Delta \mathrm{Ct})$ value method. The primers utilized for detection of EpCAM, $\beta$-catenin, and the internal reference ( $\beta$-actin) mRNA expression were as follows (van Amerongen et al., 2012): EpCAM F: 5'-GCA GGT CTA AAG CTG GTG-3', R: 5'-CCC TAT CAT CTC ACC CAT C-3'; $\beta$-catenin F: 5'-GCG TGG ACA ATG GCT ACT CAA G-3', R: 5'-CCG AGC ACG GCT AAA TCT-3'; Internal reference ( $\beta$-actin) F: 5'-TGG CAC CCA GCA CAA TGA A-3', R: 5'-CTA AGT CAT AGT CCG CTA GAA GCA-3'.

For Western blot detection, the cells were collected and washed with PBS. The protein extraction kit (AR0103, Boster, Wuhan, China) was used to extract proteins. After the extraction was completed in accordance with manufacturer instructions, the extracted proteins were quantified using standard BCA methodology. After the protein concentration was adjusted, gel electrophoresis was performed, the proteins transferred to a PVDF membrane, incubated with the abovementioned primary antibodies (EpCAM and $\beta$-catenin, 1:500, respectively), and chromogenic results were detected by chemiluminescence.

\section{Assessment of silencing efficiency and efficacy}

After successful transfection was confirmed by PCR and Western blot, the prolifera- 
tion and apoptosis of transfected or nontransfected HCT116 tumor cells were measured.

\section{Cell proliferation assessment}

The MTT assay was used for detection of cell proliferation using standard protocol. Briefly, cells in the logarithmic growth phase were cultured on a 96-well culture plate, and then incubated with MTT for 4 hours. The OD values of the cell populations were measured. Two duplicate wells were assessed for each group, and the mean value was calculated.

\section{Cell scratch test for assessment of cell motility}

Logarithmic phase cells were seeded on a 6-well plate containing $2 \mathrm{~mL}$ medium per well. The scratches were made with a $200-\mu \mathrm{L}$ sterile pipette tip through the six well plate's diameter. The scratch was made only one in each well, and can separate the cells into two parts equally. The cell movements at $6 \mathrm{~h}, 12 \mathrm{~h}$, and $24 \mathrm{~h}$ after scratching were recorded. The test was repeated 3 times, and the average value was recorded (Yoon et al., 2011).

\section{Apoptosis test}

Normal and transfected cells were cultivated to the logarithmic growth phase. When cells reached greater than $80 \%$ confluence, the cells were digested with trypsin (without EDTA), collected into an Eppendorf microtube, and the pellet harvested following centrifugation. A PI apoptosis detection kit (M3040-1, Major Bio, Shanghai, China) was used to ascertain apoptotic cells, which were quantified using flow cytometry.

\section{Statistical analysis}

The SPSS 17.0 statistical software (SPSS, Chicago, IL, USA) was used for data analyses. Data counts were analyzed by the $\chi^{2}$ test; measurement data were analyzed by the MannWhitney $t$-test, and the correlation between the numerical values was analyzed by using the Spearman correlation test, with statistically significant differences defined as $\mathrm{P}<0.05$.

\section{RESULTS}

\section{Statistical results of clinical data and follow-up outcomes}

In this study, pathological sections were collected and analyzed by immunohistochemical staining (Figure 1A-C). The results showed that the expression levels of EpCAM and $\beta$-catenin proteins in tumor tissues were significantly higher than those in para-carcinoma and normal tissues (Table 1). In different tumor tissues, the expression levels of EpCAM and $\beta$-catenin proteins had a positive correlative relationship with the grades of tumor malignancy (Table 2). After follow-up assessment of all patients with colon cancer, the results showed that the expression levels of EpCAM and $\beta$-catenin proteins in tumors were negatively correlated with patient prognoses (Table 3). 
A

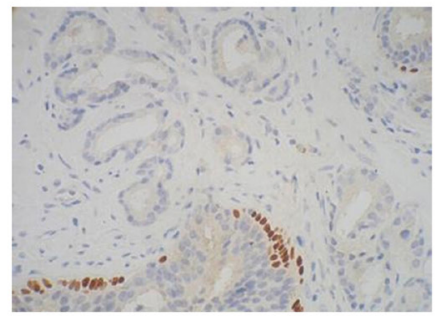

B

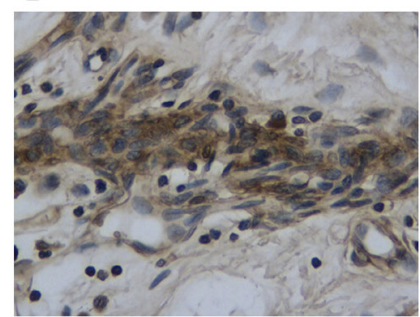

C

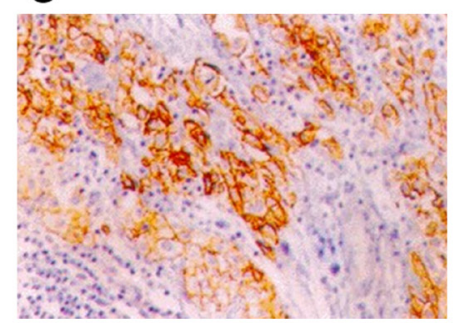

Figure 1. Schematic diagrams of immunohistochemical staining for EpCAM in clinical specimens. A. Negative staining; B. weakly positive staining; C. strongly positive staining.

Table 1. Immunohistochemical analysis of clinical pathologic sections $[\mathrm{N}=$ patients $(\%)]$.

\begin{tabular}{|c|c|c|c|c|c|c|c|c|}
\hline \multirow[t]{2}{*}{ Category } & \multicolumn{2}{|c|}{ Cancer } & \multicolumn{2}{|c|}{ Para-carcinoma tissues } & \multicolumn{2}{|c|}{ Benign lesions } & \multicolumn{2}{|c|}{ Normal tissues } \\
\hline & Negative & Positive & Negative & Positive & Negative & Positive & Negative & Positive \\
\hline$\beta$-catenin & $1(2)$ & $49(98)$ & $50(100)$ & $0(0)$ & $4(8)$ & $46(92)$ & $50(0)$ & $0(0)$ \\
\hline ЕрСAM & $4(8)$ & $46(92)$ & $45(90)$ & $5(10)$ & 47 (94) & $3(6)$ & $50(0)$ & $0(0)$ \\
\hline
\end{tabular}

Table 2. Immunohistochemical analysis of tumor tissue sections at different clinical stages $[\mathrm{N}=$ patients $(\%)]$.

\begin{tabular}{|c|c|c|c|c|c|c|}
\hline \multirow[t]{2}{*}{ Category } & \multicolumn{2}{|c|}{ Stage I } & \multicolumn{2}{|c|}{ Stage II } & \multicolumn{2}{|c|}{ Stage III } \\
\hline & Weakly positive & $\overline{\text { Strongly positive }}$ & Weakly positive & Strongly positive & Weakly positive & Strongly positive \\
\hline$\beta$-catenin & $20(87.0)$ & $3(13.0)$ & $7(36.8)$ & $12(63.2)$ & $0(0)$ & $7(100)$ \\
\hline ЕрСAM & $17(79.2)$ & $3(20.8)$ & $6(31.6)$ & $13(68.4)$ & $0(0)$ & $7(100)$ \\
\hline
\end{tabular}

Table 3. Comparison of five-year survival rates among patients with tumors [N (\%)].

\begin{tabular}{lcc}
\hline Category & Weakly positive & Strongly positive \\
\hline$\beta$-catenin & $19(70.4)$ & $9(40.9)$ \\
EpCAM & $18(78.2)$ & $10(43.5)$ \\
\hline
\end{tabular}

\section{Results of siRNA transfection}

The colon cancer cell line HCT116 was transfected by siRNA against EpCAM. PCR and western blot analysis confirmed that the mRNA and protein expression levels of EpCAM were significantly decreased in colon cancer cells after transfection (Figure 2A and B). In addition, compared to non-transfected cells, the mRNA and protein expression levels of $\beta$-catenin were also simultaneously decreased in EpCAM siRNA transfected cells (Figure 2C and D).

\section{Detection of the efficacy of siRNA-mediated EpCAM knockdown}

To determine the rate of apoptosis in tumor cells following EpCAM knockdown, the nucleic acid binding dye propidium iodide (PI) was added to untransfected and transfected tumor cells, and the number of apoptotic cells observed following flow cytometry. The results of three experimental repetitions are shown in Table 4; cell culture images are shown in Figure $3 \mathrm{~A}$ and $\mathrm{B}$. From this analysis, we found that the apoptosis ratio of transfected cells was significantly higher than that of untransfected tumor cells, suggesting that silencing of EpCAM could increase the apoptosis ratio of cells under drug stimulation. 
A

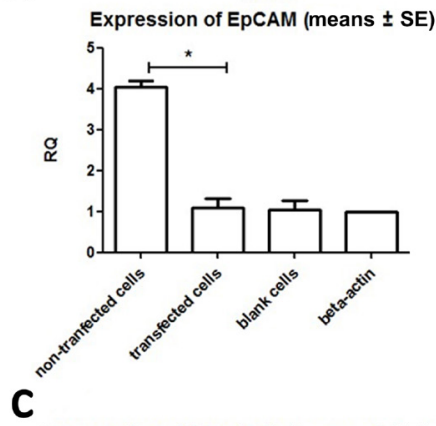

C

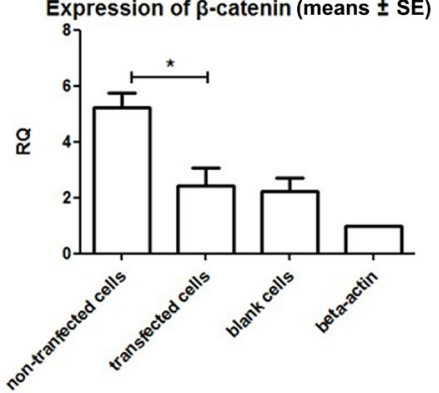

B

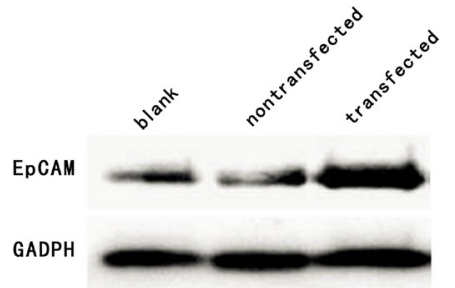

D

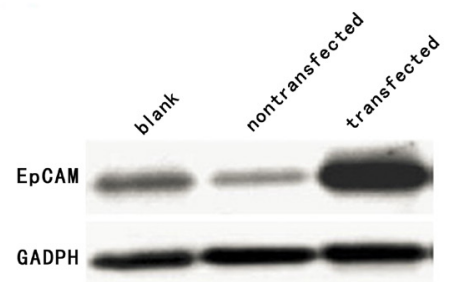

Figure 2. Comparison of EpCAM gene and protein expression between non-transfected and transfected cells. A. Polymerase chain reaction (PCR) results for EpCAM expression before and after transfection; B. western blot results for EpCAM protein expression before and after transfection; C. PCR results for $\beta$-catenin expression before and after transfection; D. western blot results for $\beta$-catenin protein expression before and after transfection.

Table 4. Apoptosis rates (Means $\pm \mathrm{SE})$.

\begin{tabular}{lccc}
\hline Grouping & $48 \mathrm{~h}$ & $72 \mathrm{~h}$ & $96 \mathrm{~h}$ \\
\hline Normal cells & $6.43 \pm 0.51$ & $13.69 \pm 2.13$ & $21.33 \pm 2.72$ \\
Transfected cells & $7.22 \pm 0.62$ & $19.36 \pm 2.18$ & $26.16 \pm 1.92$ \\
\hline
\end{tabular}

$\mathrm{SD}=$ standard deviation.

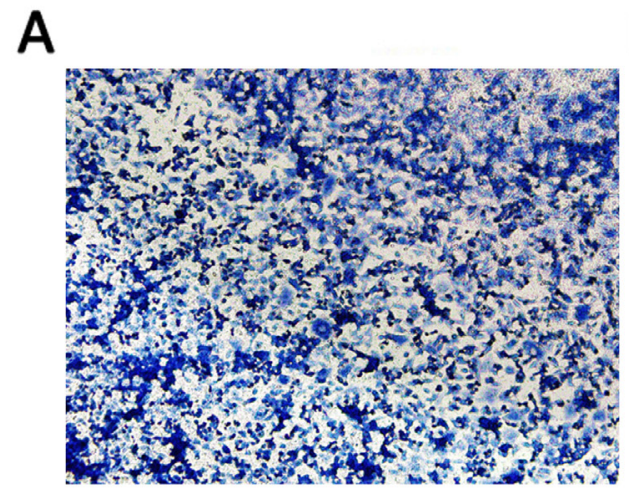

B

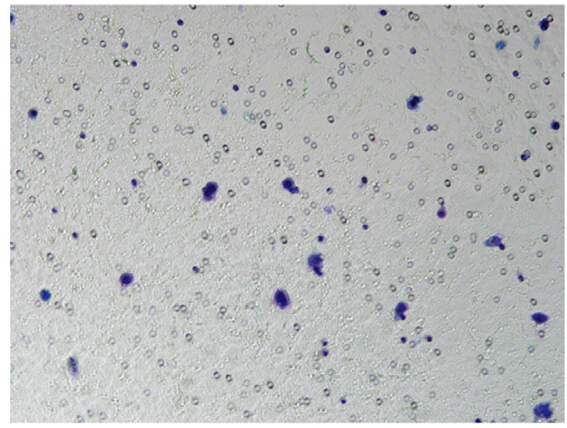

Figure 3. Comparison of the growth profiles between cells before and after transfection (Haemotoxylin staining). A. Cells before transfection; B. cells after transfection. 
Cell proliferation was ascertained using the MTT assay. OD readings were expressed as quantitative values (Table 5), which demonstrated that the proliferative activities of transfected cells were significantly decreased $(\mathrm{P}<0.05)$. These results clearly indicated that inhibition of EpCAM could lead to significant inhibition of cell proliferation. Similar results, furthermore, were also observed using the cell scratch test, wherein the scratches were filled significantly faster in the non-transfected tumor cells than in the transfected tumor cells (Table 6), implying loss of proliferative activity in the transfected cells.

Table 5. Cell proliferation activities (OD value, Means $\pm \mathrm{SE}$ ).

\begin{tabular}{lcccr}
\hline Grouping & $24 \mathrm{~h}$ & $48 \mathrm{~h}$ & $72 \mathrm{~h}$ & $96 \mathrm{~h}$ \\
\hline Normal cells & $0.16 \pm 0.002$ & $0.93 \pm 0.03$ & $1.82 \pm 0.23$ & $2.89 \pm 0.42$ \\
Transfected cells & $0.14 \pm 0.001$ & $0.65 \pm 0.05$ & $0.89 \pm 0.16$ & $1.43 \pm 0.32$ \\
\hline
\end{tabular}

$\mathrm{OD}=$ optical density; $\mathrm{SD}=$ standard deviation.

Table 6. Cell counts in the denuded areas of cell scratch tests over time.

\begin{tabular}{lccc}
\hline Grouping & $6 \mathrm{~h}$ & $12 \mathrm{~h}$ & $24 \mathrm{~h}$ \\
\hline Normal cells & $52.28 \pm 12.16$ & $121.35 \pm 22.19$ & $162.89 \pm 21.02$ \\
Transfected cells & $21.36 \pm 8.92$ & $79.28 \pm 19.18$ & $102.14 \pm 18.18$ \\
\hline
\end{tabular}

\section{DISCUSSION}

EpCAM is a tumor-associated antigen first discovered in colon cancer tissues; it is also known as CD326, KSA, and TACSTD1. The EpCAM protein consists of three structural domains: an extracellular domain, a single transmembrane domain, and an intracellular domain. The extracellular domain has been shown to assist cells expressing EpCAM with adhering to other homologous cells, and the intracellular domain can be combined with actin to interact with the cytoskeleton (Terris et al., 2010). In the physiological state, EpCAM is primarily expressed in the intercellular space of epithelial cells (except squamous epithelia), but is not expressed in other tissues such as connective tissue. However, EpCAM is widely expressed in various adenocarcinomas (Petsch et al., 2011).

The $W n t / \beta$-catenin pathway is an important pathway for regulation of the incidence and progression of tumors. Previous studies have shown that EpCAM can be involved in a Wnt pathway cascade reaction to activate proto-oncogenes such as c-myc, thus causing carcinogenic effects. In a previous clinical study of breast cancer, it has been confirmed that high EpCAM expression in breast cancer cells is closely related to the activation of the Wnt pathway (Imrich et al., 2012). It has also been reported that high EpCAM expression has a negative correlation with the prognosis of patients. The patients who have EpCAM positive cells often have poor prognoses, with concrete manifestations including a high postoperative recurrence rate and a higher lymph node metastasis rate (Mikolajczyk et al., 2011). No such results, however, are available for patients with colon cancers. This study, therefore, was designed to report the clinical characteristics of colon cancers in relation to EpCAM expression using clinical data analysis. Results indicated that in clinical samples of human colon cancer tissue, EpCAM was highly expressed in tumor tissues, but was poorly or not expressed in para-carcinoma tissues and non-tumor tissues. The differences in the distribution of EpCAM across tissue types were similar to the previously reported distribution and expression char- 
acteristics of $\beta$-catenin (Lugli et al., 2010); therefore, the authors considered that EpCAM expression might be correlated with activation of the Wnt- $\beta$-catenin pathway.

Considering the correlation between EpCAM expression and tumor prognosis observed in this study, the method of silencing EpCAM gene expression with siRNA was adopted and carried out in culture using a colon cancer cell line. After successful establishment and transfection of an EpCAM siRNA into cells, the knockdown efficiency was verified by PCR and Western blot, which confirmed effective silencing of EpCAM gene and protein expression in the colon cancer cell line. In addition, it was also observed that in the EpCAM-silenced colon cancer cells, the expression level of $\beta$-catenin was also simultaneously decreased; this result showed that silencing of EpCAM could block the activation of the Wnt- $\beta$-catenin pathway in tumor cells. Consequently, the proliferative activity and apoptosis rate of transfected cells were measured, and the results compared with those from cells that had not been transfected with siRNA. The results from these experiments showed a significant decrease in the proliferation of transfected cells and a significant increase in their apoptosis ratio. Because previous reports have acknowledged the importance of blocking the Wnt- $\beta$-catenin pathway for inhibition of tumor growth (Lu et al., 2013), the findings of this study promote further studies in this direction, and confirm that EpCAM is also an important target for the treatment of tumors, through the mechanism of blocking the $\mathrm{Wnt} / \beta$-catenin pathway.

Currently, the Wnt pathway is thought to be a classical tumor signaling pathway ( $\mathrm{Ji}$ et al., 2011). Through the analysis of clinical specimens, this study revealed the relationship of a new tumor related transmembrane protein EpCAM with the Wnt/ $\beta$-catenin pathway. This study has also provided initial confirmation of EpCAM expression in colon cancer tissues, and that patients with a high EpCAM expression level have poor prognoses. Further examination of the cellular mechanisms confirmed that the silencing of EpCAM could suppress the expression of the Wnt pathway and reduce the invasive and proliferative activities of the tumor cells. The results of this study suggest that EpCAM can be considered as a new therapeutic target in the adjuvant chemotherapy for colon cancers. In Europe, anti-EpCAM monoclonal antibody drugs have been recently used in patients with malignant ascites (Lavergne et al., 2011). In this study, the result of cell culture shows that EpCAM-targeted therapy may also have significance for the treatment of colon cancers, and it is therefore worthwhile to carry out further basic and clinical research and drug development towards this goal.

\section{ACKNOWLEDGMENTS}

Research supported by Shandong Province Science and Technology Department policy guidance projects (\#2012YD18101).

\section{REFERENCES}

Carmon KS, Gong X, Lin Q, Thomas A, et al. (2011). R-spondins function as ligands of the orphan receptors LGR4 and LGR5 to regulate Wnt/beta-catenin signaling. Proc. Natl. Acad. Sci. U. S. A. 108: 11452-11457.

Clevers H and Nusse R (2012). Wnt/beta-catenin signaling and disease. Cell 149: 1192-1205.

Imrich S, Hachmeister M and Gires O (2012). EpCAM and its potential role in tumor-initiating cells. Cell Adh. Migr. 6: 30-38.

Jawhari AU, Noda M, Farthing MJ and Pignatelli M (1999). Abnormal expression and function of the E-cadherin-catenin complex in gastric carcinoma cell lines. Br. J. Cancer 80: 322-330.

Ji J, Yamashita T and Wang XW (2011). Wnt/beta-catenin signaling activates microRNA-181 expression in hepatocellular carcinoma. Cell Biosci. 1: 4. 
Lavergne E, Hendaoui I, Coulouarn C, Ribault C, et al. (2011). Blocking Wnt signaling by SFRP-like molecules inhibits in vivo cell proliferation and tumor growth in cells carrying active beta-catenin. Oncogene 30: 423-433.

Lu H, Ma J, Yang Y, Shi W, et al. (2013). EpCAM is an endoderm-specific Wnt derepressor that licenses hepatic development. Dev. Cell 24: 543-553.

Lugli A, Iezzi G, Hostettler I, Muraro MG, et al. (2010). Prognostic impact of the expression of putative cancer stem cell markers CD133, CD166, CD44s, EpCAM, and ALDH1 in colorectal cancer. Br. J. Cancer 103: 382-390.

Mikolajczyk SD, Millar LS, Tsinberg P, Coutts SM, et al. (2011). Detection of EpCAM-negative and cytokeratin-negative circulating tumor cells in peripheral blood. J. Oncol. 2011: 252361.

Petsch S, Gires O, Ruttinger D, Denzel S, et al. (2011). Concentrations of EpCAM ectodomain as found in sera of cancer patients do not significantly impact redirected lysis and T-cell activation by EpCAM/CD3-bispecific BiTE antibody MT110. MAbs. 3: 31-37.

Spizzo G, Fong D, Wurm M, Ensinger C, et al. (2011). EpCAM expression in primary tumour tissues and metastases: an immunohistochemical analysis. J. Clin. Pathol. 64: 415-420.

Terris B, Cavard C and Perret C (2010). EpCAM, a new marker for cancer stem cells in hepatocellular carcinoma. $J$. Hepatol. 52: 280-281.

van Amerongen R, Bowman AN and Nusse R (2012). Developmental stage and time dictate the fate of Wnt/beta-cateninresponsive stem cells in the mammary gland. Cell Stem Cell. 11: 387-400.

Yoon SM, Gerasimidou D, Kuwahara R, Hytiroglou P, et al. (2011). Epithelial cell adhesion molecule (EpCAM) marks hepatocytes newly derived from stem/ progenitor cells in humans. Hepatology 53: 964-973. 